\begin{tabular}{|c|c|c|c|c|c|c|c|c|c|c|c|}
\hline \multirow{2}{*}{ 点 } & $\mathbf{J}$ & D & $\mathbf{V}$ & 1 & 2 & 2 & 9 & \multirow{2}{*}{ WILEY } & \multirow{2}{*}{ Dispatch: 9.10.13 } & Journal: JDV & CE: Banu Priva \\
\hline & Journal & Nan & & & Ianu & scril & t No. & & & No. of pages: 10 & PE: Sudhakar S \\
\hline
\end{tabular}

\title{
Detection of factor XIII-A is a valuable tool for distinguishing dendritic cells and tissue macrophages in granuloma 2 annulare and necrobiosis lipoidica
}

\author{
D. Töröcsik, ${ }^{1}$ H. Bárdos, ${ }^{2}$ Zs. Hatalyák, ${ }^{2}$ B. Dezső, ${ }^{3}$ G. Losonczy, ${ }^{4}$ L. Paragh, ${ }^{1}$ Z. Péter, ${ }^{1}$ M. Balázs, ${ }^{2}$ \\ E. Remenyik ${ }^{1}$ R. Ádány ${ }^{2, *}$ \\ ${ }^{1}$ Department of Dermatology, Faculty of Medicine, Medical and Health Science Centre, University of Debrecen, Debrecen, Hungary \\ ${ }^{2}$ Department of Preventive Medicine, Faculty of Public Health, Medical and Health Science Centre, University of Debrecen, Debrecen, \\ Hungary \\ ${ }^{3}$ Department of Pathology, Faculty of Medicine, Medical and Health Science Centre, University of Debrecen, Debrecen, Hungary \\ ${ }^{4}$ Department of Ophthalmology, Faculty of Medicine, Medical and Health Science Centre, University of Debrecen, Debrecen, Hungary \\ *Correspondence: R. Ádány. E-mail: adany.roza@sph.unideb.hu
}

\begin{abstract}
Background Factor XIII subunit A (FXIII-A) is used as a diagnostic marker in a wide range of dermatological diseases ranging from inflammatory lesions to malignancies, although neither the cell types responsible for its expression nor the mechanism(s) resulting in its local accumulation in pathological conditions have been characterized.

Objective In this study, we aimed to gain information on the cells showing an immunohistochemical reaction for FXIIIA and answer the question whether macrophages and/or dendritic cells are labelled for FXIII-A.

Methods We carried out our studies on samples of granuloma annulare (GA) and necrobiosis lipoidica (NL), the prime examples for granulomatous skin lesions with a non-infectious background in which extracellular matrix remodelling is a key feature without any sign of malignant transformation. We used markers for macrophages and dendritic cells in combination with the detection of FXIII-A in double labelling immunohistochemical reactions.

Results We demonstrated that FXIII-A positivity clearly distinguishes macrophages (CD163+/FXIII-A+) from dendritic cells (CD11C+/FXIII-A-) not only in the normal dermis as previously described by Zaba et al. (J Clin Invest 2007; 117: 2517-2525) but also in the pathological conditions of GA and NL. Detecting the expression of DC-SIGN/CD209 and mannose receptor molecules on FXIII-A+ macrophages we confirmed that FXIII-A is expressed in the alternatively activated macrophages. However, while DC-SIGN/CD209 was invariably expressed on FXIII-A+ cells both in normal and pathological conditions of GA/NL (98.7\% vs. 93.5/96\%), mannose receptor was only partially coexpressed with FXIII-A (94.8\% vs. $74.7 / 52.2 \%)$, suggesting that FXIII-A+ macrophages do not represent a homogenous population.
\end{abstract}

Conclusions Factor XIII (FXIII)-A selectively marks macrophages and distinguishes them from dendritic cells. The presence of FXIII-A is not a disease-specific marker but indicates a possible common mechanism of macrophage activation in various dermatological diseases.

Received: 19 February 2013; Accepted: 18 September 2013

\section{Conflicts of interest}

The authors state no conflict of interest.

\section{Funding sources}

This work was supported in part by National Research Funds OTKA K81847 R.A., and PD101557 (D.T.) and by the TÁMOP 4.2.2.A-11/1/KONV-2012-0031 projects. D.T. is a recipient of Janos Bolyai Fellowships of the Hungarian Academy of Sciences and Lajos Szodoray Prize of the University of Debrecen and holds a BMC grant from South Korea.

\section{Introduction}

Factor XIII (FXIII) involved in blood coagulation consists of two globular A subunits (FXIII-A) exerting a transglutaminase activity upon its activation, and two inhibitory B subunits (FXIII-B). It is well known that the activated form of FXIII-A has an important role in the final step of blood coagulation (fibrin stabilization); however, its role is not restricted to the area of haemostasis. ${ }^{1,2}$ In addition to megakaryocytes and platelets, monocytes and macrophages can also synthesize FXIII-A but are unable to secrete it. FXIII-A can be detected from the very early stage of monocyte differentiation (monoblasts in the bone marrow) via circulating blood monocytes to the macrophages of connective tissue and 
serous cavities. Based on these findings, FXIII-A is interpreted as a marker protein of the cell lines in which it acts as an intracellular transglutaminase with roles in various intracellular (intracytoplasmic and intranuclear) processes. ${ }^{3-9}$

The immunohistochemical detection of FXIII-A is widely used in dermatological pathology diagnosis, ${ }^{10-20}$ although neither the FXIII-A-positive cell types nor the possible mechanisms behind their abundant accumulation in certain lesions have been convincingly interpreted. Historically, in addition to monocyte-derived tissue histiocytes, dendritic cells, the key antigen-presenting cells of the immune system, were also considered to be positive for FXIII-A. ${ }^{10,15,18-20}$ It became widely accepted that 'FXIII-A+ cells in human skin represent a specific population of bone marrow-derived dermal dendritic cells, distinct from Langerhans cells, that share some features common to mononuclear phagocytes (monocyte/macrophages)'. ${ }^{21}$

Concerning the application of immunohistochemical detection of FXIII-A in dermatohistopathology, we face the following severe inconsistencies:

1 The presence of FXIII-A in monocyte-derived macrophages is not ubiquitous. While under pathological conditions observed in various tumours a high number of FXIII-A-positive monocyte-derived macrophages can be found, ${ }^{2-24}$ in granulomas of sarcoidosis and tuberculosis the majority of macrophages are negative for FXIII-A. ${ }^{25,26}$ To explain this phenomenon, our previous studies have confirmed that the presence of FXIII-A in macrophages indicates that they are alternatively activated through a pathway induced by the cytokines interleukin 4 (IL-4) and interleukin 13 (IL-13), whereas its absence is a trait of classically activated macrophages, in which interferon gamma (IF $\gamma$ ) is the triggering cytokine molecule. ${ }^{26}$ Dissecting the two pathways of macrophage activation upon FXIII-A positivity suggests that FXIIIA-positive macrophages are important players in conditions where extracellular remodelling is the key feature (as in tumour tissues), but not in the host vs. pathogen responses as it is in tuberculosis granulomas.

2 A recent study that aimed to distinguish macrophages from dendritic cells based on the expression of various markers and functional characteristics of these cell types in the normal human dermis illustrated that, while dermal macrophages are CD163+ FXIII-A+, dendritic cells are CD11c+ CD1c+, and these markers define discrete dermal cell populations with no overlap. ${ }^{27}$ However, this type of classification has been challenged in pathological conditions only to a limited extent. ${ }^{28}$

Our study was designed to determine whether the distinct cell populations of macrophages and dendritic cells can be clearly identified under pathological conditions upon FXIII-A positivity and whether the accumulation of FXIII-A+ cells is related to the matrix remodelling processes, a characteristic feature of the alternatively activated macrophages. For our studies, we chose to use samples of granuloma annulare (GA) and necrobiosis lipoi- dica (NL), the prime examples for dermatological diseases with a large accumulation of macrophages, in which extracellular matrix remodelling is a key feature without malignant transformation and with a non-infectious background. ${ }^{29,30}$

Here, we demonstrate that the immunohistochemical detection of FXIII-A is a suitable marker to differentiate macrophages from dendritic cells also in pathological conditions that are characterized with extracellular matrix remodelling. The characterization of FXIII-A+ cells confirmed our previous findings that FXIII-A macrophages express other markers of alternative activation, as well. In the interpretation of the development and progression of GA and NL lesions, the impressive histological appearance of foci consisting exclusively of dendritic cells surrounded by tissue compartments extremely rich in FXIII-A+ macrophages may open new avenues for research, as well.

\section{Materials and methods}

\section{Skin samples}

Skin samples were obtained from four normal volunteers who underwent plastic surgery, nine GA patients and six NL patients in the Department of Dermatology, University of Debrecen, Medical and Health Sciences Center (UDMHS). Histological diagnosis was made in the Department of Pathology, UDMHS. The acquisition of all skin specimens was reviewed and approved by the Regional and Institutional Ethics Committee, UDMHS. Informed consent was obtained, and the study was performed in accordance with the Principles of the Declaration of Helsinki.

\section{Double immunofluorescence (DIF) studies of macrophages and dendritic cells}

Frozen sections were fixed in acetone for $10 \mathrm{~min}$ and incubated in $5 \%$ normal goat serum diluted in Serum-Free Protein Block (SFPB) (DAKO, Glostrup, Denmark). Factor XIII-A was detected by rabbit affinity purified anti-human FXIII-A antibody (diluted 1 : 100, Acris Antibodies, San Diego, CA, USA) for $2 \mathrm{~h}$ at room temperature. This procedure was followed by visualization using DyLight 488 goat anti-rabbit IgG antibody for $45 \mathrm{~min}$ (diluted 1 : 40, Vector Labs, Burlingame, CA, USA). For coexpression, the detection of FXIII-A was sequentially combined with different reference markers using mouse monoclonal antihuman antibodies against CD antigens (see Table S1). Following a 10-min incubation with normal horse serum containing SFPB, the second primaries' specific binding was visualized by DyLight 549 horse anti-mouse $\operatorname{IgG}$ antibody (diluted $1: 40$, Vector Labs). Slides were washed in PBS and mounted with Vectashield Mounting Medium with DAPI (Vector Labs) to counterstain nuclei. For negative controls, the appropriate non-immune control sera [rabbit IgG from Vector Labs, mouse IgG1 or mouse IgG2b from BD Pharmingen (Heidelberg, Germany)] were used in place of primary antibodies followed by the same procedure 
as above. In general, no staining was observed; however, in some cases, non-specific background fluorescence was observed in the epidermis (not shown).

Images were acquired with an Axioplan microscope (Carl Zeiss, Oberkochen, Germany) equipped with selective filters and connected to a CCD IMAC camera (Sony, Tokyo, Japan) and ISIS fluorescent imaging system (MetaSystems, Altlussheim, Germany).

To show unambiguous colocalization, some tissue samples that were double labelled for FXIII-A and CD11c were also imaged with a confocal laser-scanning microscope (LSM 700, Carl Zeiss) equipped with solid-state lasers.

\section{Analysis of coexpression of FXIII-A with CD antigens}

On DIF samples, the degree of colocalization of FXIII-A with CD antigens was analysed semiquantitatively by counting 100 100 FXIII-A+ cells in association with other markers. Each sample was then evaluated separately, with the average percentages of cells coexpressing FXIII-A and CD antigens (Table 1 and Table S2).

\section{Results}

\section{Normal and pathological skin samples harbouring granuloma annulare and NL contain distinct FXIII-A+ and CD11c+ cell populations}

In normal skin, FXIII-A+ cells were found scattered in the papillary and reticular dermis, whereas CD11c+ cells showing no colocalization with FXIII-A+ cells were found in low numbers and mainly localized beneath the epidermis. Confirming previous data by Zaba et al., ${ }^{27}$ FXIII-A+ cells were also positive for the macrophage specific protein CD163, and coexpressed mannose receptor (MMR/CD206) and DC-SIGN/CD209 markers. On the other hand, besides the CD11c+ cells, the few cells found to express CD1a or CD1c, markers of different dendritic cell subsets, were also negative for FXIII-A (Fig. 1).

In samples of GA and NL, FXIII-A+ cells were much more abundant, and the density of CD11c+ dendritic cells was also elevated. The FXIII-A+ and CD11c+ cells showed two distinct cell populations in both skin lesions, as was observed in normal samples. Interestingly, the necrotic areas of the skin samples mainly consisted of CD11c+ cells, which were surrounded by FXIII-A+ macrophages (Fig. 2).

Regarding CD68, which is expressed in a large proportion of macrophages, a high number of $\mathrm{CD} 68+$ cells were present, but found to only partially coexpress FXIII-A. Compared with normal skin tissues, GA and NL samples exhibited more cells identified as CD1c and CD1a (Fig. 3). Very few cells were stained for the mature DC markers DC-LAMP/CD208 and/or CD83. In all cases studied, CD1c+, CD1a+, DC-LAMP/CD208+ and CD83+ cells were always negative for FXIII-A (not shown).

These results indicate that immunohistochemical profiling to separate macrophages from dendritic cells with the use of the above markers for normal skin described by Zaba et al. ${ }^{27}$ can also be applied to pathological samples including GA and NL.

\section{FXIII-A+ cells do not form a homogenous group}

The immunofluorescent data for FXIII-A coexpression with CD molecules in normal and pathological skin are listed in Table 1. In summary, the number of FXIII-A+ cells increased considerably in GA and NL skin samples. Almost the entire population of the FXIII-A+ cells showed coexpression with CD163 in normal skin samples, although the overlap of the two markers was found to be partial, but dominant in the examined granulomatous samples of GA and NL. CD68 was expressed in most of the FXIII-A+ cells in the normal skin, whereas in GA and NL only approximately $60 \%$ of the cells expressed both proteins.

Table 1 Co-expression of FXIII-A with different CD antigens in normal, granuloma annulare and necrobiosis lipoidica skin samples

\begin{tabular}{|c|c|c|c|}
\hline & \multicolumn{3}{|c|}{$\begin{array}{l}\text { Percentage of FXIII-A positive cells showing co-expression with different CD antigens (based on serial } \\
\text { sections) }\end{array}$} \\
\hline & Normal skin ( \pm SD) & Granuloma annulare $( \pm \mathrm{SD})$ & Necrobiosis lipoidica ( \pm SD) \\
\hline CD163 & $97.8( \pm 2.3)$ & $85.5( \pm 4.1)$ & $77.1( \pm 6.9)$ \\
\hline CD209 & $98.8( \pm 1.1)$ & $93.5( \pm 3.2)$ & $96.0( \pm 2.2)$ \\
\hline CD206 & $94.8( \pm 1.6)$ & $74.7( \pm 10.3)$ & $52.2( \pm 3.9)$ \\
\hline CD68 & $85.5( \pm 5.3)$ & $62.3( \pm 18.5)$ & $64.33( \pm 10.2)$ \\
\hline CD11c & 0 & $<1$ & $<1$ \\
\hline CD1c & 0 & $<1$ & 0 \\
\hline$\overline{\mathrm{CD} 1 \mathrm{a}}$ & 0 & 0 & 0 \\
\hline CD208 & 0 & 0 & 0 \\
\hline CD83 & 0 & 0 & 0 \\
\hline
\end{tabular}

Skin samples: $n=4$ normal, $n=9$ granuloma annulare, $n=6$ necrobiosis lipoidica. Numbers indicate average percent of FXIII-A+ cells labeled by antibodies against the given $\mathrm{CD}$ antigens; $\mathrm{SD}$, standard deviation. 

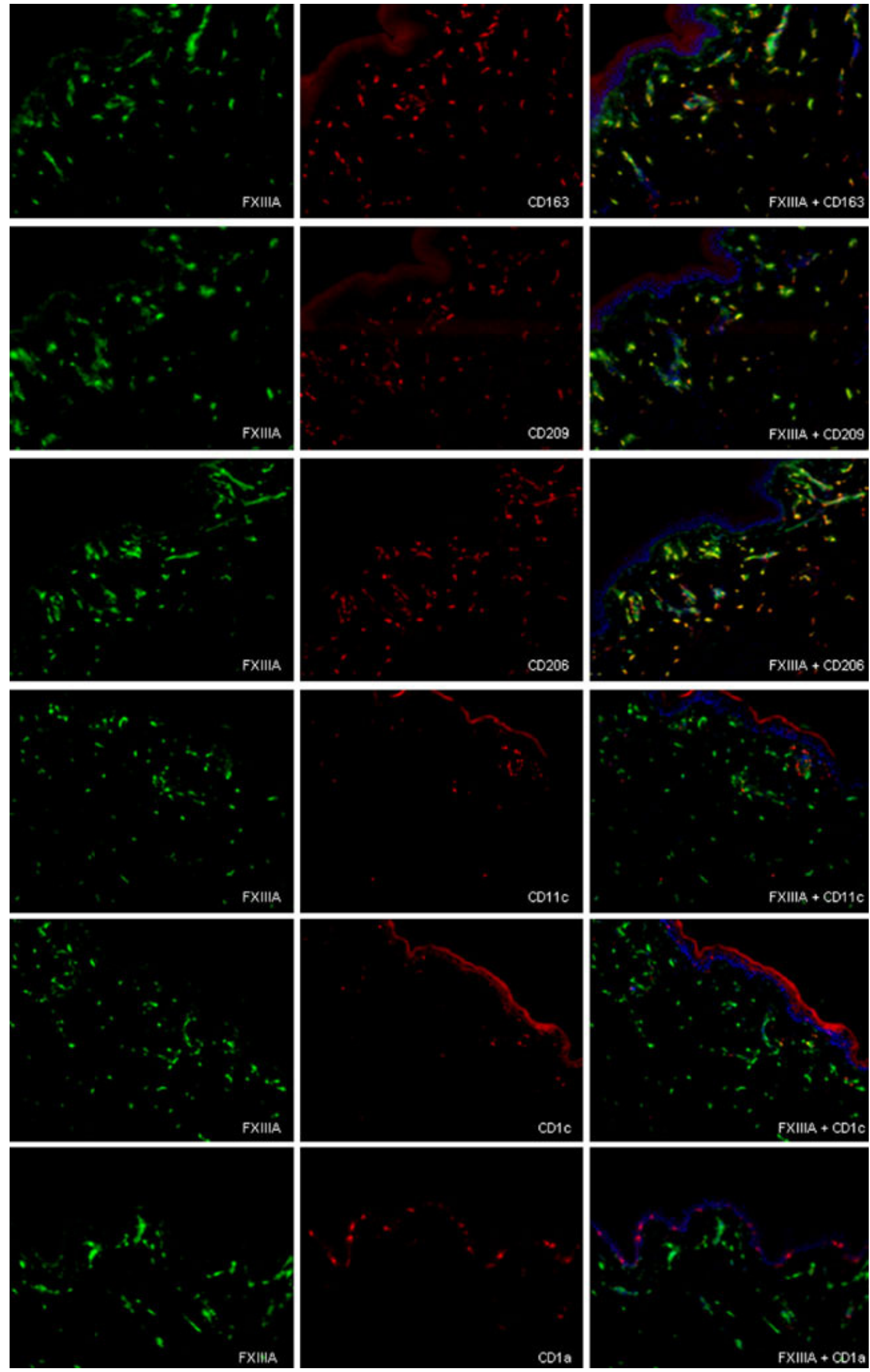
Figure 1 Distribution of macrophages and dendritic cells in normal skin. Double immunofluorescent labelling for Factor XIII (FXIII)-A (green) and CD163, DC-SIGN/CD209, CD206/MMR, CD11c, CD1c, CD1a (red) detected on serial sections (horizontal images). FXIII-A+ macrophages were labelled for CD163, CD209 and CD206 (first three rows), while CD11c-, CD1c- and CD1a-positive dendritic cells were negative for FXIII-A. In merged images, cell nuclei are counterstained with DAPI (blue). Bar $=100 \mu \mathrm{m}$.
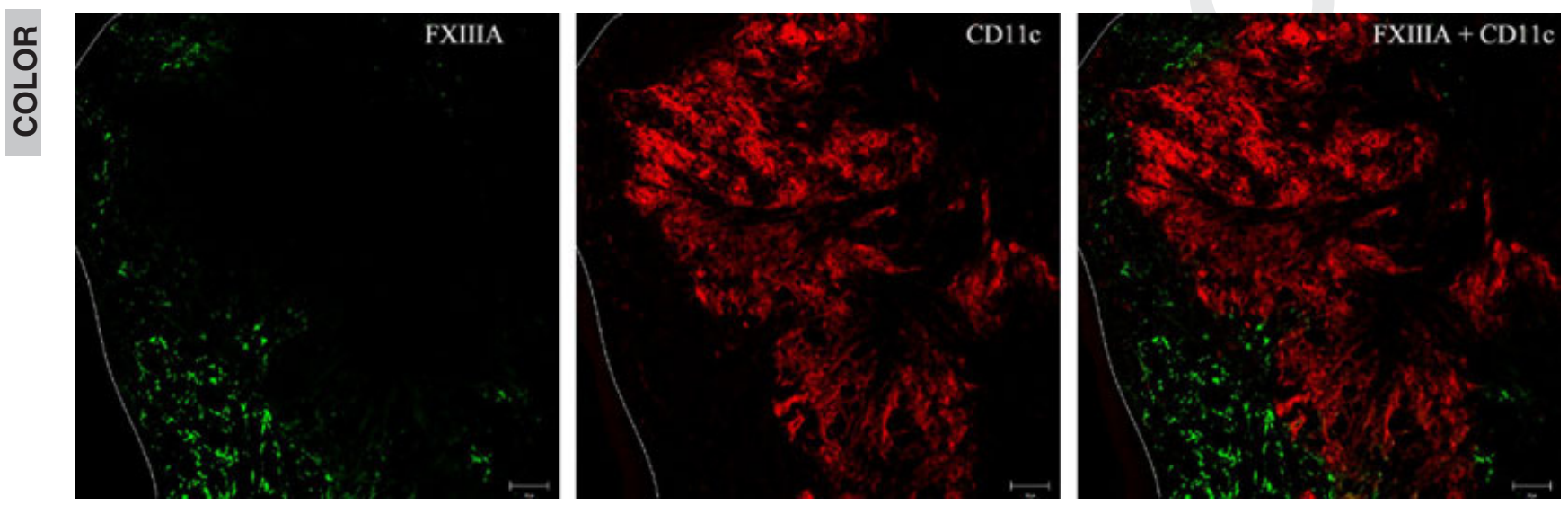

Figure 2 Factor XIII (FXIII)-A+ and CD11c+ cells represent two distinct cell populations in GA and necrobiosis lipoidica (NL) skin lesions. Double immunofluorescent labelling for FXIII-A (green) and CD11c (red), showing that both cell types were greatly accumulated in the lesions, with no overlap in the two cell populations. Note the necrotic areas mainly consisting of CD11c+ cells (red) surrounded by FXIIIA+ macrophages (green). Bar $=100 \mu \mathrm{m}$.

MMR/CD206 and DC-SIGN/CD209 expression levels were increased in IL-4-treated macrophages, just as FXIII-A was ${ }^{26}$ and were reported to be associated with the alternatively activated macrophages besides dendritic cells in experimental conditions. $^{31,32}$ Furthermore, Ochoa et al. ${ }^{33}$ showed that DC-SIGN/ CD209 expression is not restricted to DCs in the normal skin but is coexpressed with other markers of macrophages. In normal skin, almost all FXIII-A+ cells expressed CD206, but in GA and NL, approximately $75 \%$ and $50 \%$ of the FXIII-A+ cells showed coexpression of CD206 respectively. In contrast, DC-SIGN/CD209 was almost invariably expressed on FXIII-A+ cells both in normal and pathological conditions. Interestingly, the FXIII-A+ macrophages surrounding the necrotic areas also coexpressed both CD206 and DC-SIGN/CD209 (Fig. 4, Table 1 and Table S2).

This finding shows that in relation to the extracellular matrix remodelling found in GA and NL, FXIII-A+ macrophages also express other markers of alternative activation (MMR/CD206 and DC-SIGN/CD209); however, these cells do not form a homogenous population suggesting that the different expression panels mark different in vivo functions.

\section{Discussion}

Almost immediately following the detection of FXIII-A in monocytes and cultured macrophages, ${ }^{4,5}$ the immunohistochemical detection of FXIII-A was introduced into the repertoire of diagnostic dermatohistopathology. FXIII-A was detected in the stromal cells of many dermatological lesions both under inflammatory conditions (spongiotic dermatoses, lichen planus, toxic epidermal necrolysis, psoriasis, acute and chronic graft-vs.host disease), as well as in malignancies (Kaposi's sarcoma, dermatofibromas, non-Langerhans cell histiocytoses, basal cell carcinomas and malignant melanomas). ${ }^{10-20}$ The cells found to be positive for FXIII-A were interpreted in various ways (dendrocytes, histiocytes, monocyte-derived macrophages, or dendritic cells). Despite the wide application of the immunohistochemical detection of FXIII-A in dermatohistopathology, questions about why FXIII-A is expressed, what its presence indicates, and what its function might be were addressed only in limited ways, if at all.

In this study, we aimed to characterize FXIII-A+ cells in addition to normal skin in GA and NL samples and interpret our findings on the basis of already existing in vitro and in vivo data on FXIII-A+ macrophages. GA and NL were chosen for our studies as these are the two most frequently diagnosed noninfectious granulomatous diseases, in which the disruption of collagen and elastic structure results in a tissue environment that may trigger the alternative activation of macrophages. ${ }^{34,35}$

We tested one of the most intriguing questions in the application of the immunohistochemical detection of FXIII-A in the dermatohistological diagnosis, namely, whether dendritic cells, the key antigen-presenting cells of the immune system, are FXIII-A+. This was supported by observations that dendritic cells, the key antigen-presenting cells of the immune system, were also considered to be FXIII-A $+{ }^{21}$ and by findings that under in vitro conditions FXIII-A is involved in the chemotactic 

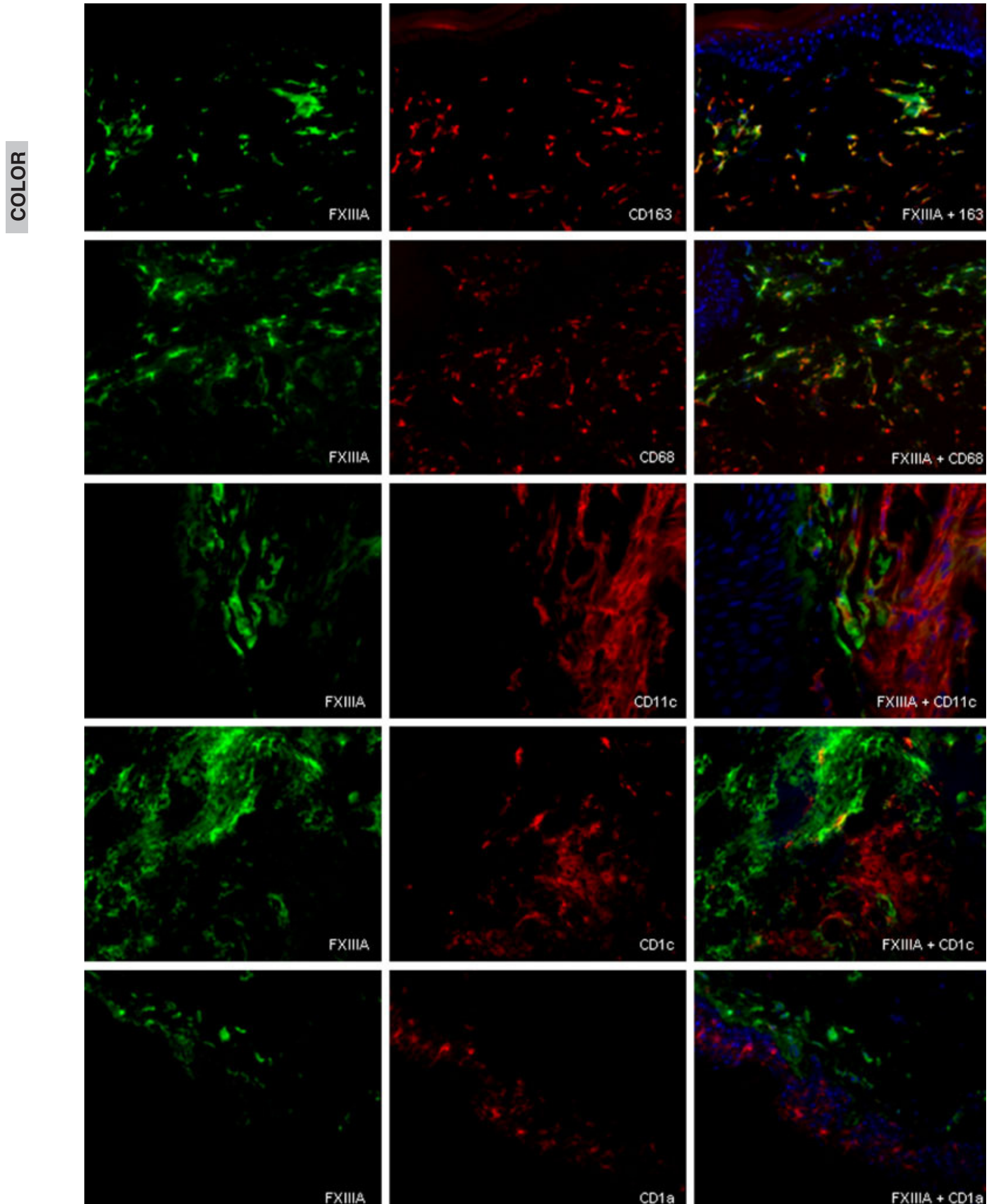
Figure 3 Distribution of marker reactions for macrophages and dendritic cells in non-infectious granulomatous lesions. Double immunofluorescent labelling for factor XIII (FXIII)-A (green) and CD163, CD68, CD11c, CD1c, CD1a (red) in granuloma annulare. FXIII-A showed coexpression with CD163, CD68, but could not be detected in CD11c-, CD1c- and CD1a-positive cells. In merged images, cell nuclei are counterstained with DAPI (blue). Bar $=50 \mu \mathrm{m}$.
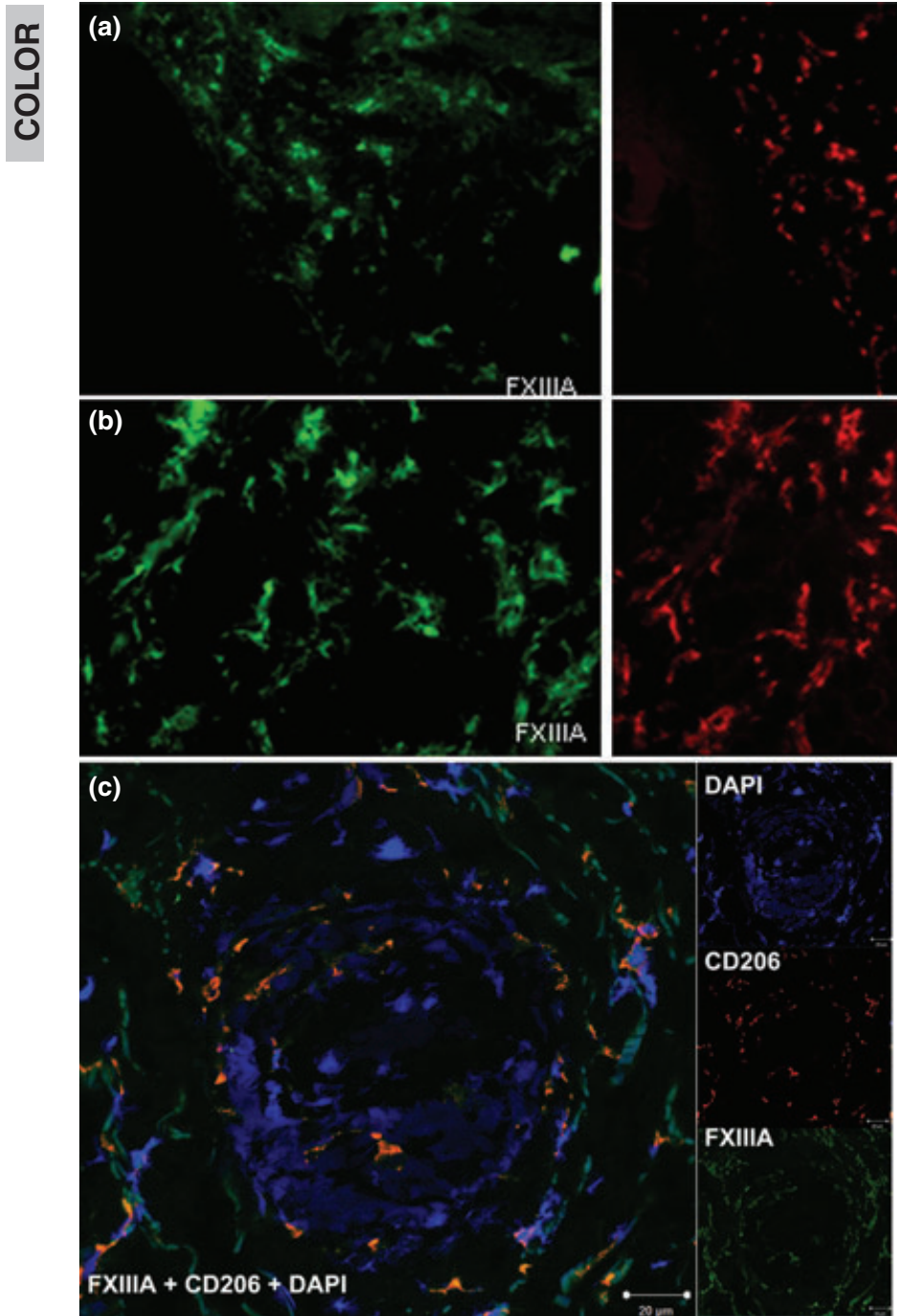
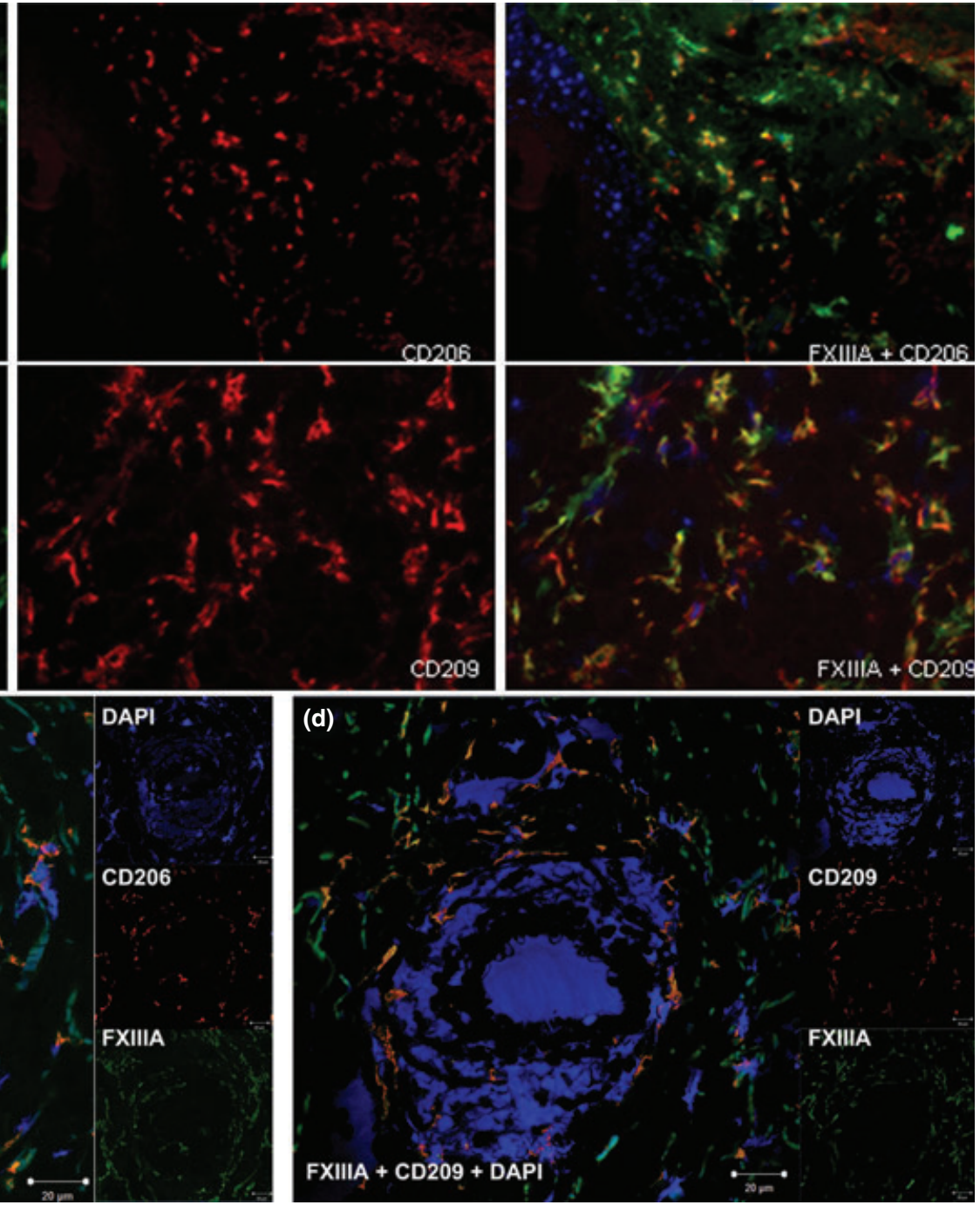

Figure 4 Factor XIII (FXIII)-A is coexpressed with markers of alternative macrophage activation (a) (b) Double immunofluorescent labelling for FXIII-A (green), MMR/CD206 and DC-SIGN/CD209 (red) in the dermis of granuloma annulare apart from the necrotic areas; (c) (d) Double immunofluorescent labelling for FXIII-A (green) and MMR/CD206 and DC-SIGN/CD209 (red), showing that FXIII-A+ macrophages surrounding the necrotic foci are positive for MMR/CD206 and DC-SIGN/CD209. In merged images, cell nuclei are counterstained with DAPI (blue). Bar $=20 \mu \mathrm{m}$.

response and migration motility of monocyte-derived dendritic cells. $^{36}$ However, Zaba et al. ${ }^{27}$ defined FXIII-A as a marker exclusive for macrophages, and suggested that previous FXIII-A positivity of dermal cells with dendritic morphology was due to a rabbit polyclonal antibody that was widely used. According to their classification, FXIII-A+ CD163+ cells (macrophages) and
CD11c+ CD1c+ cells (dendritic cells) are discrete dermal populations in the normal human dermis, or in other words, cells that express FXIII-A also express CD163 but neither CD11c nor CD1c. While FXIII-A+ CD163+ cells were unable to stimulate T cells, CD11c+ CD1c+ cells were typical antigen presenters and fulfilled all of the requirements for classifying them as dendritic 
cells. ${ }^{37}$ Regarding the interpretation of the in vitro findings on the role of FXIII-A in dendritic cell motility, ${ }^{36}$ the method used for the differentiation of monocytes into dendritic cells under in vitro conditions notably represents a combined stimulus of IL-4 and GM-CSF. ${ }^{38}$ We have previously demonstrated that IL-4 is a very potent inducer of FXIII-A expression, ${ }^{26}$ therefore the detection of FXIII-A in DCs differentiated in vitro can be considered as a direct effect of the cytokine instead of being a marker for DC differentiation.

In this study, we provide evidence that using the marker reactions mentioned above, the two cell populations characterized by Zaba et al. ${ }^{27}$ can be clearly distinguished also in pathological samples of GA and NL in which FXIII-A+ macrophages and CD11c+ dendritic cells also show distinct population.

Dissecting the cells expressing FXIII-A, we found that CD163 was present in almost the whole FXIII-A+ cell population in both the normal and the pathological samples. On the other hand CD68, another widely used macrophage marker, was found to overlap with FXIII-A only partially in the normal skin, and to a lesser extent in GA and NL samples. These findings are consistent with previous observations that besides macrophages, other cell types such as dendritic cells, granulocytes and also non-myeloid cells express CD68 under certain conditions, therefore it cannot be considered as a selective macrophage marker. ${ }^{39}$

MMR/CD206 and DC-SIGN/DC209 are markers of alternative macrophage activation; however, using these markers alone is not sufficient to detect macrophages as they are also expressed in dendritic cells. Examining their expression in combination with FXIII-A, in normal skin, FXIII+ cells expressed MMR/ CD206 and DC-SIGN/DC209. These data agree with our previous findings that FXIII-A-positive macrophages are alternatively activated in the normal human dermis. Examining the macrophages in the GA and NL samples, the coexpression of FXIII-A with DC-SIGN/DC209 was practically complete, but only partially so for MMR/DC206; nevertheless, MMR/DC206 could be observed in the majority of FXIII-A+ cells. Interestingly, in the FXIII-A+ macrophages with a pivotal role in the granuloma formation, detected around the necrotic areas mainly consisted of CD11c+ cells in the skin samples, both DC-SIGN/DC209 as well as MMR/DC206 was positive. Comparing GA and NL samples with the used marker reactions, they exerted similar macrophage populations, suggesting similar macrophage activation mechanisms in the background of the two diseases, in which matrix remodelling might be a key player. These findings and further immunohistochemical analyses of FXIII-A+ macrophages in GA and NL samples revealed that these cells do not represent a homogenous cell population. The existence of different cell clusters suggests that certain in vivo stimuli during the activation of macrophages under pathological conditions can influence their marker characteristics. On the other hand, the high number of CD11c+ DCs found in the lesions of both GA and NL suggest that despite previous histological studies where macrophages were in the focus ${ }^{30}$ to explain the underlying pathomechanisms, DCs should be taken into account as well. Based on the role of DCs in antigen presentation their accumulation likely suggests the presence of the primary antigen(s) within the lesions that trigger(s) the granuloma formation. Further analysis of the different macrophage subsets as well as a more complex analysis of the dendritic cells, the stimuli behind their accumulation and their interaction with the FXIII-A+ macrophages surrounding the necrotic foci could lead to a better understanding of their aetiology and may even provide possible therapeutic targets not only in GA and NL but also in other diseases with granuloma formation.

To draw definitive conclusions on the role of intracellular FXIII-A in dermal macrophages on the basis of its immunohistological detection is most likely not possible, but it is still interesting to speculate on the possible functions. As FXIII-A lacks a signal peptide and as there is no proof for its secretion, its extracellular activity is limited to conditions where FXIII-A is released from destroyed macrophages. ${ }^{40,41}$ Based on the findings that FXIII-A is localized mainly around the cytoplasmic vacuoles and pseudopodia and on the fact that cytoskeletal proteins such as actin, myosin and vinculin are known substrates for activated FXIII-A, FXIII-A might play important roles in certain intracellular processes in which cytoskeletal remodelling is a key feature. $^{42-45}$ Our previous studies showed that monocytes/ macrophages from FXIII-A-deficient patients exerted an impaired capacity of Fc $\gamma$ and complement receptor-mediated phagocytosis ${ }^{46}$ suggests that FXIII-A is essential in the tissue remodelling activity of macrophages both in GA and NL. The translocation of FXIII-A with a maintained transglutaminase activity to the nuclei of macrophages differentiating from monocytes has also been demonstrated, and its possible involvement in gene expression regulation was suggested. ${ }^{47}$ Comparing gene expression profiles of normal and FXIII-A-deficient cultured human macrophages by microarray revealed that FXIII-A has an important role(s) in mediating gene expression changes in macrophages during alternative activation. Clustering the genes that were differentially regulated in the absence of the functionally active FXIII-A protein showed that FXIII-A might influence immune functions, extracellular matrix remodelling and wound healing at the level of gene expression regulation. ${ }^{48}$ To support these speculations on the role of FXIII-A in the pathogenesis of GA and NL, the most important tool would be the dermatological follow-up of FXIII-A-deficient patients, but the register ${ }^{49}$ does not contain any information about these patients' dermatological diseases.

Our findings clearly demonstrate that the classification of macrophages and dendritic cells based on certain markers previously described by Zaba et al. ${ }^{27}$ is useful not only under normal skin conditions but also under pathological conditions represented by GA and NL. With this work we put forward the need for revisiting the 'traditionally' used marker reactions to identify 
macrophages and suggest the use of CD163 and CD11c to distinguish the populations of macrophages and dendritic cells under different pathological conditions. We also showed that identifying macrophages upon CD68 positivity might be misleading in the dermatopathological diagnosis, as in the examined granulomatous lesions a large population of FXIII-A macrophages were found to be negative for CD68 but not for CD163. Furthermore, we provide evidence that FXIII-A is not a disease-specific marker but indicates a possible common mechanism of macrophage activation in various dermatological diseases, which may contribute to a better understanding of disease development and progression.

\section{Acknowledgements}

We thank Imre Veres for histological verification of the diagnoses and Ágnes Bana for technical support.

\section{References}

1 Lorand L, Losowsky MS, Miloszewski KJM. Human factor XIII: fibrinstabilizing factor. Progr Haemost Thromb 1980; 5: 245-290.

2 Muszbek L, Ádány R, Mikkola H. Novel aspects of blood coagulation factor XIII I. Structure, distribution, activation, function. Crit Rev Clin Lab Sci 1996; 33: 357-421.

3 Muszbek L, Ádány R, Szegedi G et al. Factor XIII of blood coagulation in human monocytes. Thrombos Res 1985; 37: 401-410.

4 Ádány R, Belkin A, Vasilevskaya T et al. Identification of blood coagulation factor XIII in human peritoneal macrophages. Eur J Cell Biol 1985; 38: 171-173.

5 Henriksson P, Becker S, Lynch G et al. Identification of intracellular factor XIII in human monocytes and macrophages. J Clin Invest 1985; 76: 528-534.

6 Ádány R, Kiss A, Muszbek L. Factor XIII: a marker of mono- and megakariocytopoesis. Br J Haematol 1987; 67: 167-172.

7 Ádány R. Intracellular factor XIII: cellular distribution of factor XIII subunit a in humans. Semin Thromb Hamost 1996; 22: 399-408.

8 Ádány R, Bárdos H. Factor XIII subunit A as an intracellular transglutaminase. Cell Mol Life Sci 2003; 60: 1049-1060.

9 Inbal A, Muszbek L, Lubetsky A et al. Platelets but not monocytes contribute to the plasma levels of factor XIII subunit A in patients undergoing autologous peripheral blood stem cell transplantation. Blood Coagul Fibrinolysis 2004; 15: 249-253.

10 Nickoloff BJ, Griffiths CEM. Factor XIIIa-expressing dermal dendrocytes in AIDS-associated cutaneous Kaposi's sarcoma. Science 1989; 143: 1736-1737.

11 Denton KJ, Cotton DW, Wright A et al. Factor XIIIa in nodular malignant melanoma and Spitz naevi. Br J Dermatol 1990; 12: 783-786.

12 Altman DA, Nickoloff BJ. Differential expression of Factor XIIIa and CD34 in cutaneous mesenchymal tumors. J Cutan Pathol 1993; 20: 154-158.

13 Regezi JA, Daniels TE, Saeb F et al. Increased submucosal Factor XIIIa positive dendrocytes in oral lichen planus. J Oral Pathol Lab Med 1994; 13: 114.

14 Misery L, Boucheron S, Claudy AL. Factor XIIIa expression in juvenile xanthogranuloma. Acta Derma Venereol 1995; 74(Suppl): S43-S44.

15 Fivenson DP, Nickoloff BJ. Distinctive dendritic cell subsets expressing factor XIIIa, CDla, CD1b and CD1c in mycosis fungoides and psoriasis. J Cutan Pathol 1995; 22: 223-228.

16 Goldblum JR, Tuthill RJ. CD34 and factor-XIIIa immunoreactivity in dermatofibrosarcoma protuberans and dermatofibroma. Am J Dermatopathol 1997; 19: 147-153.

17 Moretto JC, Soslow R, Smoller BR. Atypical cells in radiation dermatitis express Factor XIIIa. Am J Dermatopathol 1998; 20: 370-372.
18 Fullen DR, Headington JT. Factor XIIIa-positive dermal dendritic cells and HLA-DR expression in radial versus vertical growth-phase melanomas. J Cutan Pathol 1998; 25: 553-558.

19 Jardim Criado RF, Sotto MN et al. . Dermal dendrocytes FXIIIA+ phagocytizing extruded mast cell granules in drug-induced acute urticaria. J Eur Acad Dermatol Venereol 2013; 27: 105-112.

20 Deguchi M, Aiba S, Ohtani $\mathrm{H}$ et al. Comparison of the distribution and numbers of antigenpresenting cells among T-lymphocyte-mediated dermatoses: CD1a+, factor XIIIa+, and CD68+ cells in eczematous dermatitis, psoriasis, lichen planus and graft-versus-host disease. Arch Dermatol Res 2002; 294: 297-302.

21 Cerio R, Griffiths CE, Cooper KD et al. Characterization of factor XIIIa positive dermal dendritic cells in normal and inflamed skin. Br J Dermatol 1989; 121: 421-431.

22 Ádány R, Muszbek L. Cells containing factor XIII subunit a in benign and malignant soft tissue tumours. Histopathology 1987; 11: 1341-1343.

23 Bardos H, Molnar P, Csecsei G et al. Fibrin deposition in primary and metastatic human brain tumours. Blood Coagul Fibrinolysis 1996; 7: 536-548.

24 Bardos H, Juhasz A, Repassy G et al. Fibrin deposition in squamous cell carcinomas of the larynx and hypopharynx. Thromb Haemost 1998; 80: 767-772.

25 Probst-Cousin S, Poremba C, Rickert CH et al. Factor XIIIa expression in granulomatous lesions due to sarcoidosis or mycobacterial infection. Pathol Res Pract 1997; 193: 741-745.

26 Töröcsik D, Bárdos H, Nagy L et al. Identification of factor XIII-A as a marker of alternative macrophage activation. Cell Mol Life Sci 2005; 62: 2132-2139.

27 Zaba LC, Fuentes-Duculan J, Steinman RM et al. Normal human dermis contains distinct populations of CD11c+BDCA-1+ dendritic cells and CD163+FXIII-A+ macrophages. J Clin Invest 2007; 117: 2517-2525.

28 Fuentes-Duculan J, Suárez-Fariñas M, Zaba LC et al. A subpopulation of CD163-positive macrophages is classically activated in psoriasis. J Invest Dermatol 2010; 130: 2412-2422.

29 Stefanaki K, Tsivitanidou-Kakourou T, Stefanaki C et al. Histological and immunohistochemical study of granuloma annulare and subcutaneous granuloma annulare in children. J Cutan Pathol 2007; 34: 392-396.

30 Müller CS, Hiatt KM, Vogt T et al. Expression of CD163 in granulomatous dermatitis is not the tool that makes the difference. J Eur Acad Dermatol Venereol 2012; 26: 793-795.

31 Stein M, Keshav S, Harris $\mathrm{N}$ et al. Interleukin 4 potently enhances murine macrophage mannose receptor activity: a marker of alternative immunologic macrophage activation. J Exp Med 1992; 176: 287-292.

32 Puig-Kröger A, Serrano-Gómez D, Caparrós E et al. Regulated expression of the pathogen receptor dendritic cell-specific intercellular adhesion molecule 3 (ICAM-3)-grabbing nonintegrin in THP-1 human leukemic cells, monocytes, and macrophages. J Biol Chem 2004; 279: 25680-25688.

33 Ochoa MT, Loncaric A, Krutzik SR et al. "Dermal dendritic cells" comprise two distinct populations: CD1+ dendritic cells and CD209+ macrophages. J Invest Dermatol 2008; 128: 2225-2231.

34 Martinez FO, Helming L, Gordon S. Alternative activation of macrophages: an immunologic functional perspective. Annu Rev Immunol 2009; 27: 451-483.

35 Mantovani A, Sozzani S, Locati M et al. Macrophage polarization: tumor-associated macrophages as a paradigm for polarized M2 mononuclear phagocytes. Trends Immunol 2002; 23: 549-555.

36 Jayo A, Conde I, Lastres P et al. Possible role for cellular FXIII in monocyte-derived dendritic cell motility. Eur J Cell Biol 2009; 8: 423-431.

37 Zaba LC, Krueger JG, Lowes MA. Resident and "inflammatory" dendritic cells in human skin. J Invest Dermatol 2009; 129: 302-308.

38 Sallusto F, Lanzavecchia A. Efficient presentation of soluble antigen by cultured human dendritic cells is maintained by granulocyte/macrophage colony-stimulating factor plus interleukin 4 and downregulated by tumor necrosis factor alpha. J Exp Med 1994; 179: 1109-1118.

39 Gottfried E, Kunz-Schughart LA, Weber A et al. Expression of CD68 in non-myeloid cell types. Scand J Immunol 2008; 67: 453-463. 
40 Kaetsu H, Hashiguchi T, Foster D et al. Expression and release of the a and b subunits for human coagulation factor XIII in baby hamster kidney (BHK) cells. J Biochem 1996; 119: 961-969.

41 Katona E, Nagy B, Kappelmayer J et al. Factor XIII in bronchoalveolar lavage fluid from children with chronic bronchoalveolar inflammation. J Thromb Haemost 2005; 3: 1407-1413.

42 Cohen I, Blankenberg TA, Border D et al. Factor XIIIa-catalyzed crosslinking of platelet and muscle actin: regulation by nucleotides. Biochim Biophys Acta 1980; 628: 365-375.

43 Cohen I, Young-Bandala L, Blankenberg TA et al. Fibrinoligase-catalyzed crosslinking of myosin from platelet and skeletal muscle. Arch Biochem Biophys 1979; 192: 100-111.

44 Asijee GM, Muszbek L, Kappelmayer J et al. Platelet vinculin: a substrate of activated factor XIII. Biochim Biophys Acta 1988; 954: 303-308.

45 Muszbek L, Bereczky Z, Bagoly Z et al. Factor XIII: a coagulation factor with multiple plasmatic and cellular functions. Physiol Rev 2011; 91: 931972.

46 Sárváry A, Szücs S, Balogh I et al. Possible role of factor XIII subunit A in Fcy and complement receptor-mediated phagocytosis. Cell Immunol 2004; 228: 81-90.

47 Ádány R, Bárdos H, Antal M et al. Factor XIII of blood coagulation as a nuclear crosslinking enzyme. Thromb Haemost 2001; 85: 845-851.
48 Töröcsik D, Szeles L, Paragh G Jr et al. Factor XIII-A is involved in the regulation of gene expression in alternatively activated human macrophages. Thromb Haemost 2010; 104: 709-717.

49 Seitz R, Duckert F, Lopaciuk S et al. ETRO Working Party on Factor XIII questionnaire on congenital factor XIII deficiency in Europe: status and perspectives study group. Semin Thromb Hemost 1996; 22: 415418 .

\section{Supporting information}

Additional Supporting Information may be found in the online version of this article:

Table S1. Antibodies used in the study.

Table S2. Co-expression of FXIII-A in association with CD antigens was analyzed semi-quantitatively by counting 100 100 FXIII-A+ cells on DIF samples from nine patients with granuloma annulare (GA1-GA9), six patients with necrobiosis lipoidica (NL1-NL6) and four samples from normal skin (NORM1-NORM4). 


\section{Author Query Form}

\section{Journal: JDV}

Article: $\quad 12290$

Dear Author,

During the copy-editing of your paper, the following queries arose. Please respond to these by marking up your proofs with the necessary changes/additions. Please write your answers on the query sheet if there is insufficient space on the page proofs. Please write clearly and follow the conventions shown on the attached corrections sheet. If returning the proof by fax do not write too close to the paper's edge. Please remember that illegible mark-ups may delay publication.

Many thanks for your assistance.

\begin{tabular}{|l|l|l|}
\hline Query reference & Query & Remarks \\
\hline 1 & $\begin{array}{l}\text { AUTHOR: If there are fewer than } 7 \text { authors for all References, please supply all of } \\
\text { their names. If there are } 7 \text { or more authors, please supply the first 3 author names } \\
\text { then et al. Please check and update all such references found in the list. }\end{array}$ \\
\hline 2 & $\begin{array}{l}\text { AUTHOR: Please note that no additional changes will be accepted after your correc- } \\
\text { tions are submitted. }\end{array}$ \\
\hline
\end{tabular}


Required software to e-Annotate PDFs: Adobe Acrobat Professional or Adobe Reader (version 8.0 or above). (Note that this document uses screenshots from Adobe Reader $\mathrm{X}$ )

The latest version of Acrobat Reader can be downloaded for free at:http://get.adobe.com/reader/

Once you have Acrobat Reader open on your computer, click on the Comment tab at the right of the toolbar:

닙

This will open up a panel down the right side of the document. The majority of tools you will use for annotating your proof will be in the Annotations section, pictured opposite. We've picked out some of these tools below:

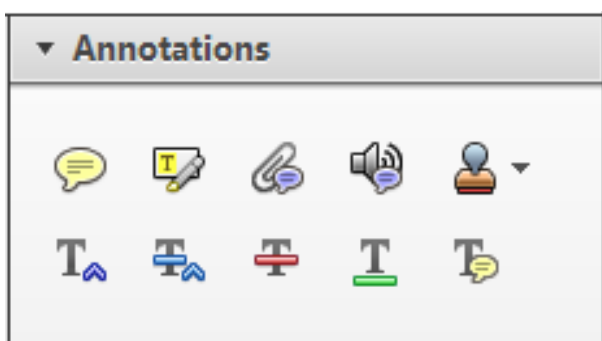

1. Replace (Ins) Tool - for replacing text.

\section{Strikes a line through text and opens up a text} box where replacement text can be entered.

\section{How to use it}

- Highlight a word or sentence.

- Click on the Replace (Ins) icon in the Annotations section.

- Type the replacement text into the blue box that appears.

Idard tramework for the analysis of $\mathrm{m}$ icy Nevertheless, it also led to exog،

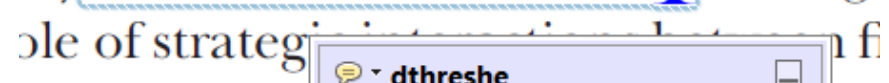
aber of comp 08/06/2011 15:58:17 $\quad$ o

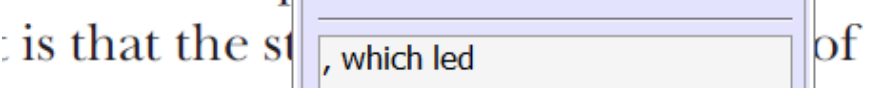
nain compo: be level, are exc nc

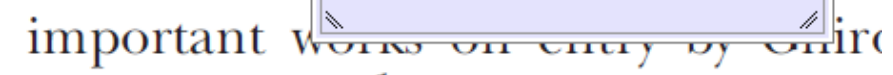
M heneforth) ${ }^{1}$ we anen the 'hlarl $\mathrm{h}$

3. Add note to text Tool - for highlighting a section to be changed to bold or italic.

Th Highlights text in yellow and opens up a text box where comments can be entered.

\section{How to use it}

- Highlight the relevant section of text.

- Click on the Add note to text icon in the Annotations section.

- Type instruction on what should be changed regarding the text into the yellow box that annears.

namic responses of mark ups ent with the VAR evidence

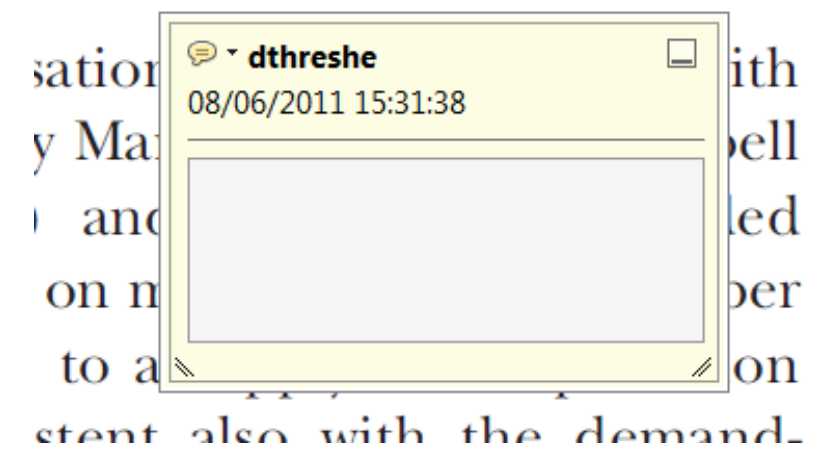

2. Strikethrough (Del) Tool - for deleting text.

Thikes a red line through text that is to be deleted.

\section{How to use it}

- Highlight a word or sentence.

- Click on the Strikethrough (Del) icon in the Annotations section.

there is no room for extra protits al c ups are zero and the number of ret) values are not determined by Blanchard and Kiyetaki (1987), rfect competition in general equilil ts of aggregate demand and supply lassical framework assuming monol eon an evorenois number of firms

\section{Add sticky note Tool - for making notes at} specific points in the text.

\section{Marks a point in the proof where a comment} needs to be highlighted.

\section{How to use it}

- Click on the Add sticky note icon in the Annotations section.

- Click at the point in the proof where the comment should be inserted.

- Type the comment into the yellow box that appears.

lallu allu suppiy silucks. Iviusl ul

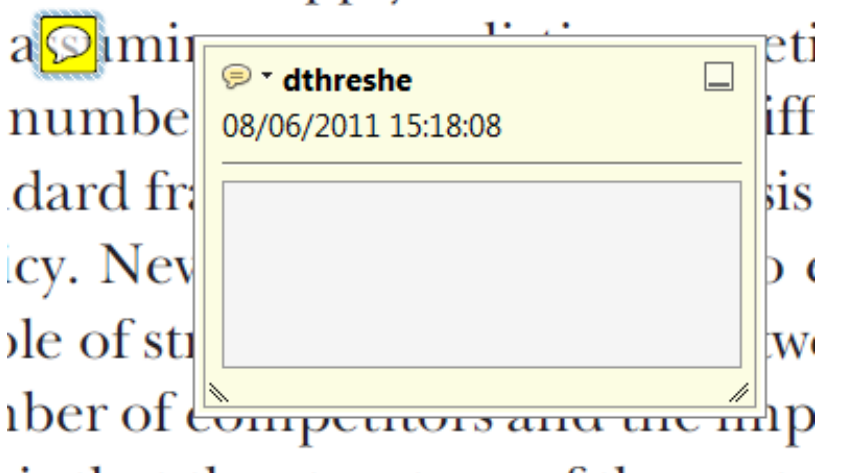

is that the structure of the secto 
5. Attach File Tool - for inserting large amounts of text or replacement figures.

Inserts an icon linking to the attached file in the appropriate pace in the text.

How to use it

- Click on the Attach File icon in the Annotations section.

- Click on the proof to where you'd like the attached file to be linked.

- Select the file to be attached from your computer or network.

- Select the colour and type of icon that will appear in the proof. Click OK.

E N D

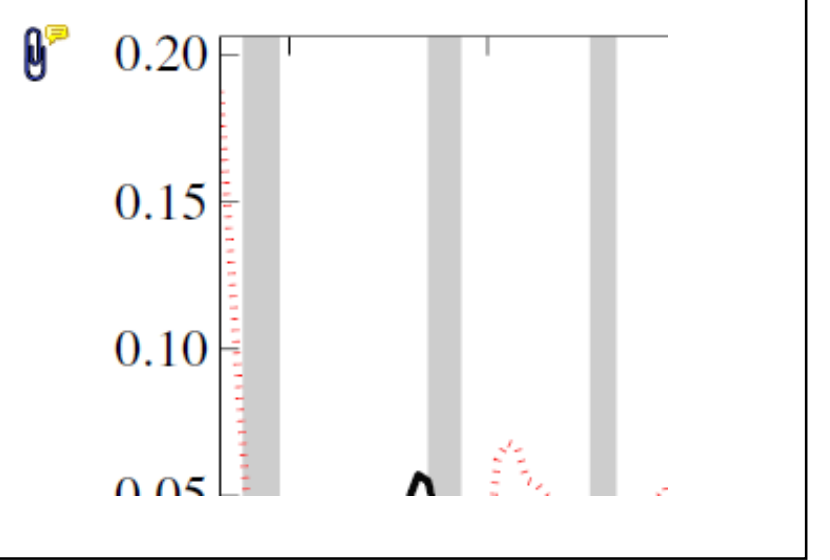

6. Add stamp Tool - for approving a proof if no corrections are required.

- Inserts a selected stamp onto an appropriate place in the proof

\section{How to use it}

- $\quad$ Click on the Add stamp icon in the Annotations section.

- Select the stamp you want to use. (The Approved stamp is usually available directly in the menu that appears).

- Click on the proof where you'd like the stamp to appear. (Where a proof is to be approved as it is, this would normally be on the first page).

of the Dusiness cycie, starting with the on perfect competition, constant ret

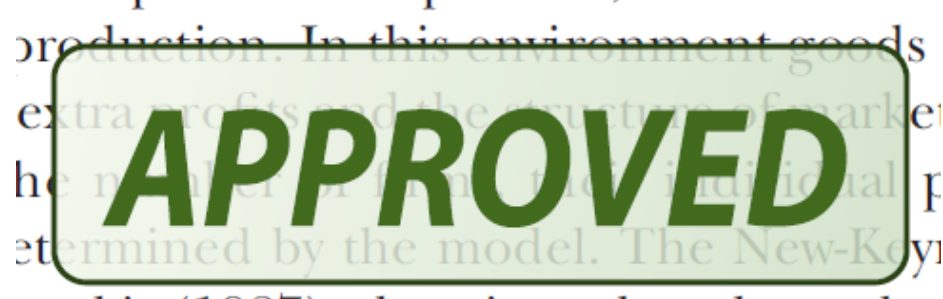

otaki (1987), has introduced produc general equilibrium models with nomin
- Drawing Markups

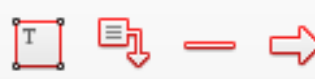

$0 \square \sqrt{6} D$

\section{How to use it}

- Click on one of the shapes in the Drawing Markups section.

- Click on the proof at the relevant point and draw the selected shape with the cursor.

- To add a comment to the drawn shape, move the cursor over the shape until an arrowhead appears.

- Double click on the shape and type any text in the red box that appears.
7. Drawing Markups Tools - for drawing shapes, lines and freeform annotations on proofs and commenting on these marks.

Allows shapes, lines and freeform annotations to be drawn on proofs and for comment to be made on these marks..

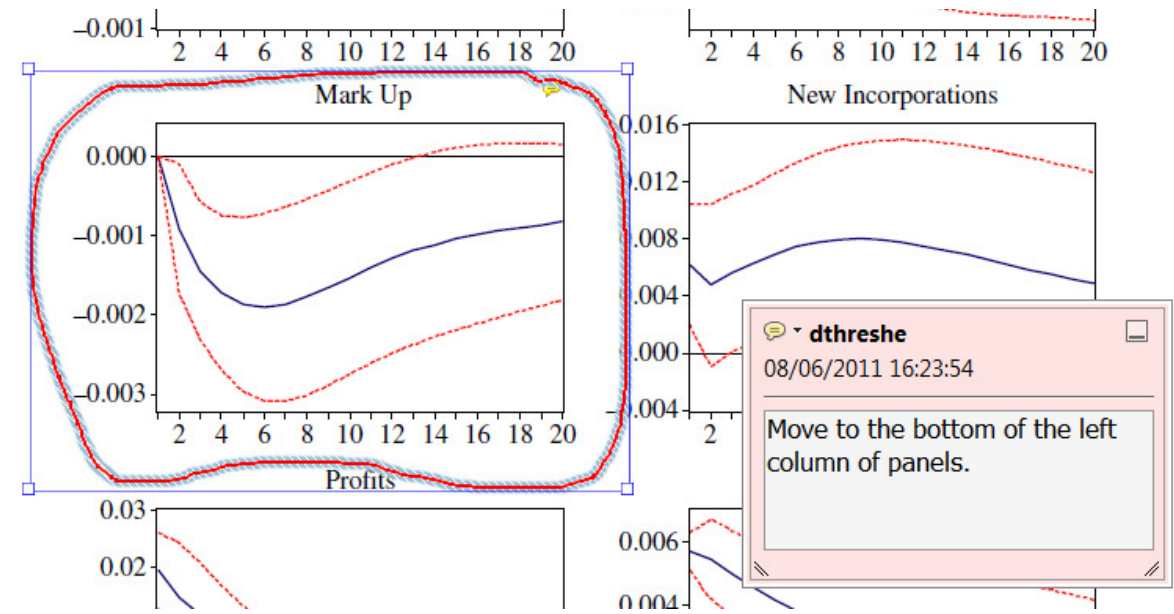

For further information on how to annotate proofs, click on the Help menu to reveal a list of further options:

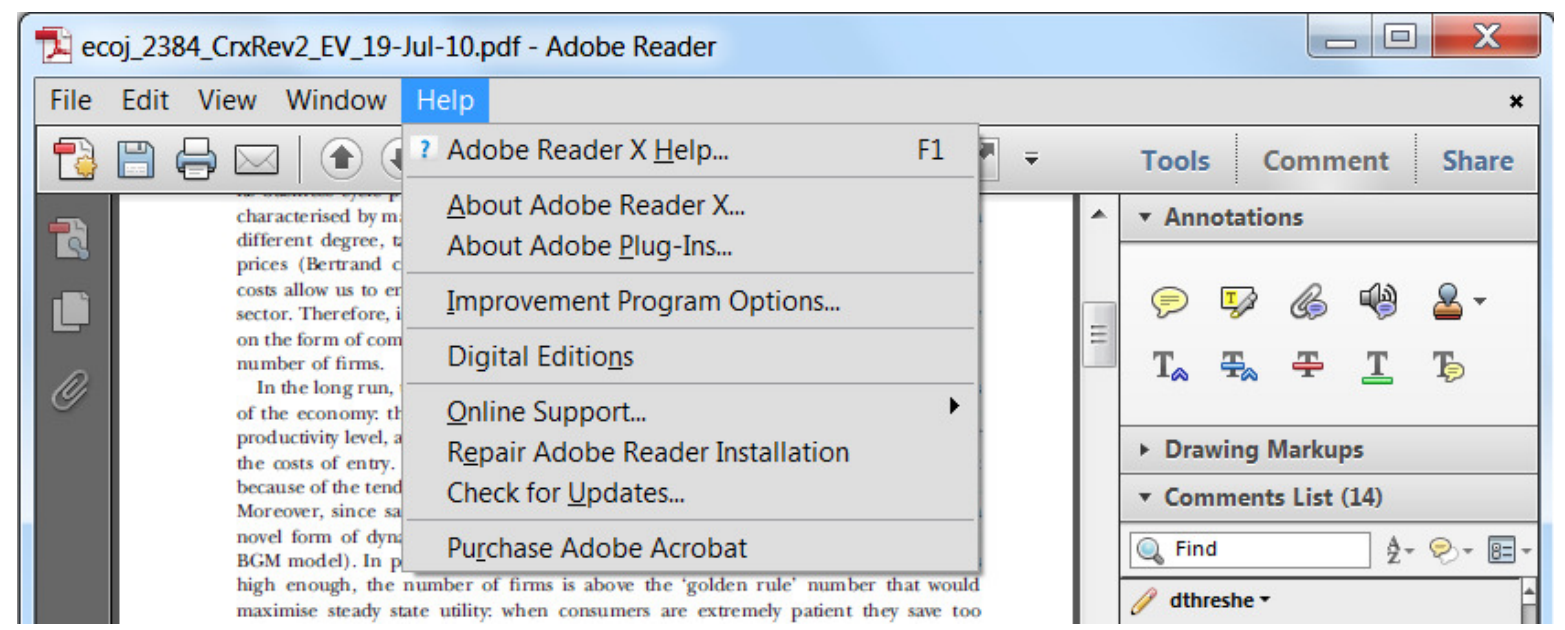

